\title{
Pipes Construction Based on the Pipes' Centre Curve Shapes
}

\author{
Kusno $^{1}$ \\ ${ }^{1}$ Department of Mathematics, University of Jember, Indonesia \\ Correspondence: Kusno, Department of Mathematics, Jl. Kalimantan No.37 University of Jember, Indonesia. E-mail: \\ kusno.fmipa@unej.ac.id
}

Received: June 23, 2019 Accepted: July 24, 2019 Online Published: July 28, 2019

doi:10.5539/jmr.v11n4p69

URL: https://doi.org/10.5539/jmr.v11n4p69

\begin{abstract}
Parts of pipeline need a long piece, a short segment, and various inflate-deflate models. They require as well the thickness and curvature of the pipes. The objective of this paper is to obtain some formulas for modeling the long pipe, the short tube, and various inflate-deflate pipe patches. Relating to the purposes, we use their cross-section, longitudinal section, and center curves of the pipe parts. The methods are, the using of the polar coordinates and of the real functions, to define the cross and longitudinal section of the pipe patches, respectively. Then, we calculate three orthonormal vectors that are determined by the tangent vectors of the pipe center curves and two unit vectors that are perpendicular to the tangent vectors. After that, we evaluate the formulas to model the long pipes and the short pipes, both inflate-deflate and thickness shapes. The results show that, using its center curves of the pipe, it is handy to design the long and short pipes, multiple thicknesses, various volume fluctuations of the pipes, and useful to model the inflate-deflate pipe parts.
\end{abstract}

Keywords: center curve, construction, cubic-quartic Bézier, Hermite curve, inflate-deflate, pipe shapes, thickness

\section{Introduction}

There were presented some methods to define the pipeline models. Lü \& Pottmann (1996) introduced an algorithm for computing the pipe surfaces of rational spine curve (center curve of pipe) that can admit a rational parameterization. Maekawa, et al. (1998) investigated the pipe surface intersection both local and global self-intersection. Then, using the unification of two cylinders of revolution defined by different radii, Malaček \& Šibrava (2006) constructed the circular surfaces. Research by Moon (2009) studied the curvature effect of pipes spin curve. He used the radial distance function form to compensate for the distortion of the pipes. Meanwhile, we can present the geometric properties of canal surfaces in which their spine curves are in the form of a unit circle and a straight line (Öztürk et al., 2010). Also, we can evaluate the substantial form of transitional tube parts whose cross sections have a polygonal shape and made of materials that cannot be creased or prolonged (Obradovic et al., 2012). This geometrical theory is based on the classical line geometry, and it can be straightforwardly implemented to the conception of a computer algorithm that generates the middle surfaces between two polygons. Fok et al. (2012) introduced the method for reconstruction of disjoint surfaces with a single equation from sectional data. Using a parametric design system of modular Pipe- $Z$ and its parametrization can be found a trefoil, a gure-eight knot, and a pentafoil (Zawidski \& Nishinari, 2013). To provide good tamper localization accuracy can be designed a topology verification for drawings the piping isometric that is in robust versus local similarity displacement and invariant to the elongation process on pipes (Su et al., 2015). The study by Bizzarri et al. (2015) presented an algebraic condition to give the guarantees that a canal surface possesses rational generalized contour, then, Moritani et al. (2018) proposed a new method to built a piping system through cylinder-based registration and model fitting of laser-scanned point clouds. On the other hand, to multiple pipes having arbitrary posses and free form cross-section, Zhou \& Qian (2013) used the constructive $\mathrm{G}^{1}$ pipes connection. Meanwhile, Bhatt et al. (2015) computed with disjoint B-spline surface. Furthermore, Kusno \& Cahyo (2018) constructed a pipeline by using the continuous connection between adjacent pipe parts through the curve of the pipes center of a line, Bézier, and Hermite forms.

In general, the introduced methods of pipes construction use a moving trihedron formula that is defined by their spin curves. Because of its curve curvatures formula, it is useful to design the short pipes of constant radius, but, they lack in constructing and connecting the tubes of long pieces. Departing from these limitations, we will present a new formula that offers to design the long pipes with its surfaces of inflate-deflate form. Then, we evaluate the moving trihedron formula to obtain the short pipes of various thickness and inflate-deflate shapes.

This article is structured in the following steps. In the first step, we will discuss the mathematics equations to construct the pipe pieces. Based on a tangent vector of pipes center curve that is defined by the cubic, quartic Bézier and the cubic Hermite curves, we introduce a formula to designing the pipes of long pieces. In the third step, we present the 
construction of short pipe parts having the thickness and inflate-deflate shapes. Finally, we summarize and wrap up the results.

\section{Mathematics Equations of Pipe Pieces}

The pipe cross section shapes can be defined by the parametric curves $\mathbf{P}(v)$ in the plane $\left[\mathbf{v}_{1}, \mathbf{v}_{2}\right]$ with $\mathbf{v}_{1}$ and $\mathbf{v}_{2}$ two orthonormal vectors as follows (Kusno, 2019).

$$
\mathbf{P}_{1}(v)=r(v)\left(\cos \varphi \mathbf{v}_{1}+\sin \varphi \mathbf{v}_{2}\right)
$$

with $\varphi=2 \pi v$ and $0 \leq v \leq 1$. In this case we choose $r(v)$ in the form

$$
\begin{array}{ll}
r_{l}(v)=p \pm q \cdot \cos \varphi ; & r_{2}(v)=p \pm q \cdot \sin \varphi ; \\
r_{3}(v)=p \cos (n \varphi) ; & r_{4}(v)=p \sin (n \varphi) ;
\end{array}
$$

with the rose leafs number $n$ and $p, q$ positive real constants. On the other hand, we can represent the various exterior and interior cross-section boundaries of the pipe by using equation (1) and or the formula

$$
\mathbf{P}_{2}(v)=r_{5}(v) \cdot\left[\cos \left((2 i+1) \cdot \frac{\pi}{n}-\frac{\varphi}{n}\right) \mathbf{v}_{1}+\sin \left((2 i+1) \cdot \frac{\pi}{n}-\frac{\varphi}{n}\right) \mathbf{v}_{2}\right]
$$

with $r_{5}(v)=r_{o} \cdot\left[\cos \left((2 i+1) \cdot \frac{\pi}{n}-\frac{\varphi}{n}\right)\right] \pm \sqrt{\left[r_{o}^{2} \cdot \cos ^{2}\left((2 i+1) \cdot \frac{\pi}{n}-\frac{\varphi}{n}\right)-\left(r_{o}^{2}-\tau^{2}\right)\right]}$ for $i=0,1, \ldots, n-1$ and $\varphi=2 \pi v$ with radius of circles $\mathrm{r}_{0}, \tau$ in the condition $\left(\mathrm{r}_{0}^{2}-\tau^{2}\right)>0$ and $0 \leq v \leq 1$.

We can formulate the boundary curve shapes of pipe in the longitudinal direction by using the real function $\rho(u)$ with $0 \leq$ $u \leq 1$ of the trigonometric function, cubic Bézier, cubic and quartic Hermite respectively in the form (Kusno, 2018; Kusno, 2010; Mortenson, 1996; Lischultz, 1969)

$$
\begin{gathered}
\rho_{\mathrm{T}}(u)=p+q \cos (2 \pi u)+r \sin (2 \pi u) \\
\rho_{\mathrm{B}}(u)=P_{o}(1-u)^{3}+3 P_{1}(1-u)^{2} u+3 P_{2}(1-u) u^{2}+P_{3} u^{3} \\
\rho_{\mathrm{H} 3}(u)=\rho_{\mathrm{H} 3}(0) H_{1}(u)+\rho_{\mathrm{H} 3}(1) H_{2}(u)+\rho_{H 3}^{u}(0) H_{3}(u)+\rho_{H 3}^{u}(1) H_{4}(u) \\
\rho_{H 4}(u)=q(u)=q_{o}\left[a u^{4}+(2-2 a) u^{3}+(a-3) u^{2}+1\right]+q_{x}\left[b u^{4}-2 b u^{3}+b u^{2}\right]+ \\
q_{1}\left[c u^{4}-(2+2 c) u^{3}+(c+3) u^{2}\right]+q_{o}^{u}\left[d u^{4}+(1-2 d) u^{3}+(d-2) u^{2}+u\right]+q_{1}^{u}\left[e u^{4}+(1-2 e) u^{3}+(e-1) u^{u}\right]
\end{gathered}
$$

with

$$
\begin{gathered}
a=\frac{-\left(2 u_{x}^{3}-3 u_{x}^{2}+1\right)}{u_{x}^{4}-2 u_{x}^{3}+u_{x}^{2}} ; \quad b=\frac{1}{\left(u_{x}^{4}-2 u_{x}^{3}+u_{x}^{2}\right)} ; \quad c=\frac{\left(2 u_{x}^{3}-3 u_{x}^{2}\right)}{\left(u_{x}^{4}-2 u_{x}^{3}+u_{x}^{2}\right)} ; \quad d=\frac{-\left(u_{x}^{3}-2 u_{x}^{2}+u_{x}\right)}{\left(u_{x}^{4}-2 u_{x}^{3}+u_{x}^{2}\right)} ; \quad e=\frac{\left(-u_{x}^{3}+u_{x}^{2}\right)}{\left(u_{x}^{4}-2 u_{x}^{3}+u_{x}^{2}\right)} ; \\
H_{1}(u)=2 u^{3}-3 u^{2}+1 ; \quad H_{2}(u)=2 u^{3}+3 u^{2} ; \quad H_{3}(u)=u^{3}-2 u^{2}+u ; \quad H_{4}(u)=u^{3} .
\end{gathered}
$$

The values $p, q, r$ are real constants, $(q+r)<p$ and $0 \leq u \leq 1$. The real value $\rho_{\mathrm{H}}(0), \rho_{\mathrm{H}}(1), \rho_{H}^{u}(0), \rho_{H}^{u}(1)$ and the Bézier control points $P_{i}$ for $i=0,1, \ldots, 3$ must be determined. The values $\rho_{H}^{u}(0)$ and $\rho_{H}^{u}(1)$ are respectively the tangent line gradient of graph at the point $\left(0, \rho_{\mathrm{H}}(0)\right)$ and $\left(1, \rho_{\mathrm{H}}(1)\right)$.

As an illustration, if the data $r(v)$ is $r(v)=<6-6 \cdot \sin (2 \pi v),(6-6 \cdot \sin (2 \pi v)) \cdot \cos (2 \pi v)$, $5+(6-6 \cdot \sin (2 \pi v)) \cdot \sin (2 \pi v)\rangle, \quad r(v)=\langle 0, \quad 1.25 \cdot(\cos (2 \pi v)) \pm 4.5, \quad 1.25 \cdot(\sin (2 \pi v))+3.75\rangle, \quad r(v)=\langle 0$, $\cos (2 \pi v) \pm 2.75, \sin (2 \pi v)-4.5\rangle, r(v)=\langle 0,4 \cdot \cos (2 \pi v), 4 \cdot \sin (2 \pi v)\rangle$, then we will find the pipe cross-section shapes in Figure 1a. In case $r_{5}(v)$ of the equation (2) determined by $\mathrm{r}_{0}=12, \mathrm{n}=8$ and $\tau=5$, we can combine it with the function $r(v)=\langle 0,(5.05) \cdot \cos (8 \pi v)+\sin (14 \pi v) \cdot \cos (2 \pi v),(5.05) \cdot \cos (8 \pi v)+\sin (14 \pi v) \cdot \sin (2 \pi v)\rangle$, then the result shapes are shown in Figure 1b. When the real function $\rho_{\mathrm{B}}(u)$ is $\rho_{\mathrm{B}}(u)=2 .(1-u)^{3}+3.7(1-u)^{2} u+3.1(1-u) u^{2}+5 u^{3}$ and the function $\rho_{\mathrm{T}}(u)$ is $\rho_{\mathrm{T}}(u)=2+0.5 \cos (\pi u)-\sin (2 \pi u)$, we will obtain respectively the graph of the pipe's longitudinal shapes in Figure 1c and Figure 1d.

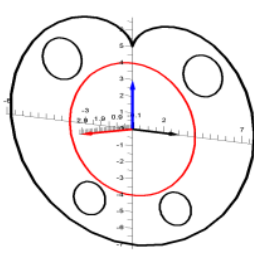

(a)

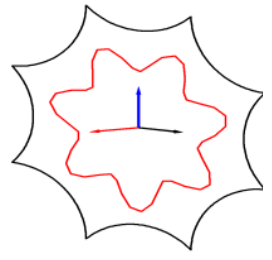

(b)

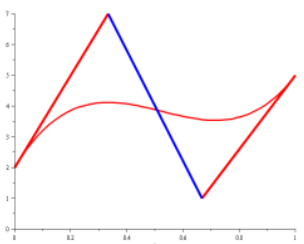

(c)

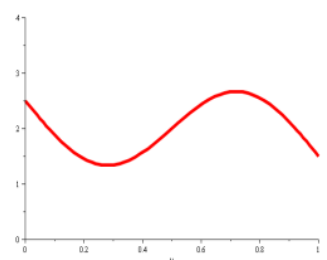

(d)

Figure 1. Some models of the pipe cross-section forms (a), (b), and longitudinal section forms (c), (d) 
Let a regular curve $\Gamma(u)$ be differentiated twice, continuous on the interval $0 \leq u \leq 1$. Furthermore, in the natural parameter $\mathrm{s}$, we consider that, $\mathbf{t}$, and $\mathbf{n}$ are the tangent, and the normal unit vector of each point and

$$
\begin{gathered}
\mathbf{t}=\frac{d \Gamma}{d s}=\dot{\Gamma}=\frac{\Gamma^{\prime}}{\left|\Gamma^{\prime}\right|} \\
\mathbf{n}=-\frac{\mathbf{k}}{|\mathbf{k}|}
\end{gathered}
$$

with $\mathbf{k}$ in the form

$$
\mathbf{k}=\frac{d \mathbf{t}}{d s}=\dot{\mathbf{t}}=\frac{\mathbf{t}^{\prime}}{\left|\Gamma^{\prime}\right|} \text {. }
$$

Thus, the unit binormal vector $\mathbf{b}$ can be formulated by $\mathbf{b}=\mathbf{t} \wedge \mathbf{n}$ that form the moving trihedron $\mathbf{t}, \mathbf{n}$, and $\mathbf{b}$ (Mortenson, 1996; Kreyzig, 1991; Lischultz, 1969; Carmo, 1976).

Consider a regular parametric curve segment $\mathbf{C}(u)$ of class $C^{2}$. In the normal plane $[\mathbf{b}, \mathbf{n}]$, we can define the circles in which their radius is $\Upsilon_{C}(u, v)=r(v) \rho(u)$, its centers are on the curve segment $\mathbf{C}(u)$, and they are orthogonal to the vector $\mathbf{t}$ along the direction of parameter $0 \leq u \leq 1$. Therefore, for $\varphi=2 \pi v$, we can construct the tube patch relatively to the vectors $[\mathbf{b}, \mathbf{n}]$ as follows

$$
\mathbf{T}_{1}(u, v)=\mathbf{C}(u)+\Upsilon_{C}(u, v)(\cos \varphi \mathbf{b}+\sin \varphi \mathbf{n}) .
$$

To avoid a singularity, we must have that the normal unit vector $\mathbf{n}$ of the curve segment $\mathbf{C}(u)$ must be only in the same sign (orientation) in the interval domain $0 \leq u, v \leq 1$.

According to Equation (6a), we can introduce a new formulation of pipe that depend on the tangent vector value $\mathbf{t}$ of the regular curve $\mathbf{C}(u)$ by using calculations as follows. Consider the triple orthonormal vectors in space $\left[\mathbf{v}_{1}, \mathbf{v}_{2}, \mathbf{t}\right]$ with $\mathbf{t}$ non zero tangent vectors of curve $\mathbf{C}(u)$ in the form Equation (6a). If at every value $u$, the tangent vector $\mathbf{t}$ is $\mathbf{t}_{u}=\left\langle t_{x}, t_{y}, t_{z}\right\rangle \neq \mathbf{0}$, then we can determine the vectors $\mathbf{v}_{2}=\left\langle v_{2 x}, v_{2 y}, v_{2 z}\right\rangle$, and $\mathbf{v}_{1}=\left\langle v_{1 x}, v_{1 y}, v_{1 z}\right\rangle$ with $\mathbf{t} \cdot \mathbf{v}_{2}=t_{x} \cdot v_{2 x}+t_{y} \cdot v_{2 y},+t_{z} \cdot v_{2 z}=0$, and the vector $\mathbf{v}_{1}=\mathbf{t} \wedge \mathbf{v}_{2}$. To define the vector $\mathbf{v}_{2}$, first, we determine a constant reference vector $\mathbf{v}_{0}$ that is not in line with the vector $\mathbf{t}$ and in every value $u$ the vector $\left(\mathbf{C}(u)-\mathbf{v}_{0}\right) \neq \mathbf{0}$. In the plane $\left[\left(\mathbf{C}(u)-\mathbf{v}_{0}\right), \mathbf{t}(u)\right]$, the projection of the vector $\left(\mathbf{C}(u)-\mathbf{v}_{0}\right)$ to the tangent vector $\mathbf{t}(u)$ is $\mathbf{p}=\left[\left(\mathbf{C}(u)-\mathbf{v}_{0}\right) \cdot \mathbf{t}(u)\right] \mathbf{t}(u)$. Thus, we can find the vector $\mathbf{w} \perp \mathbf{t}$ in the form $\mathbf{w}=\left[\left(\mathbf{C}(u)-\mathbf{v}_{0}\right)-\mathbf{p}\right]$ $\neq \mathbf{0}$, and the unit vector $\mathbf{v}_{2}$ can be determined by the formula

$$
\mathbf{v}_{2}=\frac{\mathbf{w}}{\|\mathbf{w}\|}=\frac{\left[\left(\mathbf{C}(u)-\mathbf{v}_{0}\right)-\mathbf{p}\right]}{\left\|\left(\mathbf{C}(u)-\mathbf{v}_{0}\right)-\mathbf{p}\right\|} .
$$

If $\mathbf{v}_{2} \perp \mathbf{t}$ and $\mathbf{v}_{1}=\mathbf{t} \wedge \mathbf{v}_{2}$ are defined, then, for $\varphi=2 \pi v$, the tube patches in Equation (8) can be replaced by another formula

$$
\mathbf{T}_{2}(u, v)=\mathbf{C}(u)+\Upsilon_{C}(u, v)\left[\cos \varphi \mathbf{v}_{1}+\sin \varphi \mathbf{v}_{2}\right] .
$$

Pipeline construction, in general, requires long pieces of pipe than short pieces. Modeling the long pipe pieces are, usually, used to design a variety of pipe shapes and piping systems, while the short pieces are to handle the connections between the pipes. For these reasons, this paper, first, more discusses Equation (10) to construct various models of tubes rather than Equation (8). Second, relating to Equation (8) and their application to pipes' geometric connection $\mathrm{G}^{2}$ between the short pipe pieces (Kusno and Anton 2018), graphically, we will test the effect of choosing the Equations $(2,3,4)$ and their parameters that determine the shapes of pipe both cross-section and/or longitudinal profiles. Due to Kusno (2019) has evaluated the pipes' center curve $\mathbf{C}(u)$ in Equation (8) of a line form, we will develop the curves $\mathbf{C}(u)$ in the cubic Bézier, the quartic Bézier, and Hermite curve types.

\section{Pipes Construction}

\subsection{Pipes Construction Using Center Curves of Cubic, Quartic Bézier and Cubic Hermite Curves}

Consider that the regular curves $\mathbf{C}(u)$ are respectively in cubic and quartic Bézier curves

$$
\begin{gathered}
\mathbf{C}_{3}(u)=\mathbf{B}_{3}(u)=\mathbf{P}_{0}(1-u)^{3}+3 \mathbf{P}_{1}(1-u)^{2} u+3 \mathbf{P}_{2}(1-u) u^{2}+\mathbf{P}_{3} u^{3} \\
\mathbf{C}_{4}(u)=\mathbf{B}_{4}(u)=\mathbf{P}_{0}(1-u)^{4}+4 \mathbf{P}_{1}(1-u)^{3} u+6 \mathbf{P}_{2}(1-u)^{2} u^{2}+4 \mathbf{P}_{3}(1-u) u^{3}+\mathbf{P}_{4} u^{4}
\end{gathered}
$$

with $0 \leq u \leq 1$, and $\mathbf{P}_{\mathrm{o}}, \mathbf{P}_{1}, \mathbf{P}_{2}, \mathbf{P}_{3}$, and $\mathbf{P}_{4}$ are the control points of the Bézier curves. Then the first derivative of the cubic and quartic Bézier curves are respectively

$$
\begin{gathered}
\mathbf{B}_{3}{ }^{\prime}(\mathrm{u})=3\left[\left(\mathbf{P}_{1}-\mathbf{P}_{\mathrm{o}}\right)(1-u) \cdot(1-u)+2\left(\mathbf{P}_{2}-\mathbf{P}_{1}\right)(1-u) \cdot u+\left(\mathbf{P}_{3}-\mathbf{P}_{2}\right) \cdot u^{2}\right]=\left\langle\mathrm{R}_{x 3}(\mathrm{u}), \mathbf{R}_{y 3}(\mathrm{u}), \mathbf{R}_{z 3}(\mathrm{u})\right\rangle \\
\mathbf{B}_{4}{ }^{\prime}(\mathrm{u})=4\left[\left(\mathbf{P}_{1}-\mathbf{P}_{\mathrm{o}}\right)(1-u)^{3}+3\left(\mathbf{P}_{2}-\mathbf{P}_{1}\right)(1-u)^{2} \cdot u+3\left(\mathbf{P}_{3}-\mathbf{P}_{2}\right)(1-u) \cdot u^{2}+\left(\mathbf{P}_{4}-\mathbf{P}_{3}\right) u^{3}\right]=\left\langle\mathbf{R}_{x 4}(\mathrm{u}), \mathbf{R}_{y 4}(\mathrm{u}), \mathbf{R}_{z 4}(\mathrm{u})\right\rangle
\end{gathered}
$$

with $R_{x 3}(u)=3\left[\left(P_{1 x}-P_{o x}\right) .(1-u) \cdot(1-u)+2\left(P_{2 x}-P_{1 x}\right)(1-u) \cdot u+\left(P_{3 x}-P_{2 x}\right) \cdot u^{2}\right], \quad R_{y 3}(u)=3\left[\left(P_{1 y}-P_{o y}\right) .(1-u) \cdot(1-u)+2\left(P_{2 y}\right.\right.$ - 
$\left.\left.P_{1 y}\right)(1-u) \cdot u+\left(P_{3 y}-P_{2 y}\right) \cdot u^{2}\right]$, and $R_{z 3}(u)=3\left[\left(P_{1 z}-P_{o z}\right) \cdot(1-u) \cdot(1-u)+2\left(P_{2 z}-P_{1 z}\right)(1-u) \cdot u+\left(P_{3 z}-P_{2 z}\right) \cdot u^{2}\right]$.

Using Equation (6a) can determine the tangent unit vector $\mathbf{t}_{B 3}$ and $\mathbf{t}_{B 4}$ as follows

$$
\begin{aligned}
\mathbf{t}_{B 3} & =\left(1 / \mathrm{s}_{3}\right)\left\langle R_{x 3}(u), R_{y 3}(u), R_{z 3}(u)\right\rangle \\
\mathbf{t}_{B 4} & =\left(1 / \mathrm{s}_{4}\right)\left\langle R_{x 4}(u), R_{y 4}(u), R_{z 4}(u)\right\rangle
\end{aligned}
$$

with $\mathrm{s}_{3}=\left[R_{x 3}{ }^{2}(u)+R_{y 3}{ }^{2}(u)+R_{z 3}{ }^{2}(u)\right]^{1 / 2}$ and $\mathrm{s}_{4}=\left[R_{x 4}{ }^{2}(u)+R_{y 4}{ }^{2}(u)+R_{z 4}{ }^{2}(u)\right]^{1 / 2}$.

On the other hand, given the reguler curves $\mathbf{C}(u)$ of cubic Hermite curve

$$
\mathbf{H}(u)=\mathbf{H}_{o} H_{1}(u)+\mathbf{H}_{1} H_{2}(u)+\mathbf{H}_{o}^{u} H_{3}(u)+\mathbf{H}_{1}^{u} H_{4}(u)
$$

with $H_{1}(u), H_{2}(u), H_{3}(u)$ and $H_{4}(u)$ the base functions in Equation (5b). The vectors $\mathbf{H}_{o}=\mathbf{H}(0) ; \mathbf{H}_{1}=\mathbf{H}(1)$; $\mathbf{H}_{o}^{u}=\mathbf{H}^{\prime}(0) ; \mathbf{H}_{1}^{u}=\mathbf{H}^{\prime}(1)$ and $0 \leq \mathrm{u} \leq 1$. Then the first derivative of the curve is

$$
\mathbf{H}^{\prime}(u)=\mathbf{H}_{o}\left(6 u^{2}-6 u\right)+\mathbf{H}_{1}\left(-6 u^{2}+6 u\right)+\mathbf{H}_{o}^{u}\left(3 u^{2}-4 u+1\right)+\mathbf{H}_{1}^{u}\left(3 u^{2}-2 u\right)=\left\langle H_{x}(u) ; H_{y}(u) ; H_{z}(u)\right\rangle \text {. }
$$

Thus, we obtain the tangent unit vector of the curve

with $h=\left[H_{x}^{2}(u)+H_{y}^{2}(u)+H_{z}^{2}(u)\right]^{1 / 2}$.

$$
\mathbf{t}_{H}=1 / h\left\langle H_{x}(u) ; H_{y}(u) ; H_{z}(u)>\right.
$$

Based on Equations (11) up to (16), and Equation (10), the construction procedure of pipe patches can be undertaken as follows.

a. Determine a regular parametric curve $\mathbf{C}(u)$ of class $C^{2}$, after that, select Equations $(2 \mathrm{a}, 2 \mathrm{~b}, 3)$ to define the pipe cross-section shapes, and Equations $(4 \mathrm{a}, 4 \mathrm{~b}, 4 \mathrm{c}, 4 \mathrm{~d})$ to model the pipe longitudinal such that $Y_{C}(u, v)$ is defined. In this step, the formulated pipe radius $r_{C}(u, v)$ may be constant or various measure along the curve $\mathbf{C}(u)$.

b. Choose a constant vector $\mathbf{v}_{0}$ that meets $\left(\mathbf{C}(u)-\mathbf{v}_{0}\right) \neq \mathbf{0}$ and $\mathbf{v}_{0} \neq \mathrm{kt}$ with $\mathrm{k}$ a real scalar.

c. Calculate the unit vector $\mathbf{v}_{2}$ in Equation (9) with $\mathbf{p}=[\mathbf{C}(u) \cdot \mathbf{t}(u)] \mathbf{t}(u)$ in which the vectors $\mathbf{C}(u)$ and $\mathbf{t}(u)$ are respectively one of the curves and its tangent vectors of Equations (11a,13a), (11b,13b ) or $(14,16)$.

d. Compute the unit vector $\mathbf{v}_{1}=\mathbf{t} \wedge \mathbf{v}_{2}$ and the criteria of Equation (10) is met.

In this procedure, to ensure that the surfaces of modeled pipes in Equation (10) are not twisted, we must evaluate the needed criteria of the determined center curve $\mathbf{C}(u)$ and the length of the tubes radius $Y_{C}(u, v)$. For these reasons, we will discuss the case $\mathbf{C}(u)$ of a plane curve, after that, we study the case $\mathbf{C}(u)$ of a space curve.

\subsubsection{Case: $\mathbf{C}(u)$ Plane Curves}

First, we place a regular curve $\mathbf{C}(u)$ respectively of cubic, quartic Bézier or cubic Hermite curves in a plane $\alpha$. Then, we determine $Y_{C}(u, v)$ of constant radius (Figure $2 \mathrm{~h}$ ) or inflate-depfate radius (Figure $2 \mathrm{~d}, \mathrm{e}, \mathrm{f}, \mathrm{g}$ ) and the elected vector $\mathbf{v}_{0}$ is met $\left(\mathbf{C}(u)-\mathbf{v}_{0}\right) \neq \mathbf{0}$ and $\mathbf{v}_{0} \neq \mathrm{kt}$ with $\mathrm{k}$ a real scalar for all values $u$ in the domain $0 \leq u \leq 1$. After that, the vectors $\mathbf{v}_{2}$ of Equation (9) can be computed for every $u$ in interval $0 \leq u \leq 1$ and the unit vector $\mathbf{v}_{1}=\mathbf{t} \wedge \mathbf{v}_{2}$ can be calculated uniquely. To obtain the cross-section simmetricity shape of pipes, when $Y_{C}(u, v)$ define the inflate-deflate pipe, we must lay the constant vector $\mathbf{v}_{0}$ and the curve $\mathbf{C}(u)$ in the same plane $\alpha$.

Second, if we choose the shapes of the curves $\mathbf{C}(u)$ that are fluctuative in direction $u$ in the plane $\alpha$ (Figure 2a), then we necessary to detect, locally, wether, for $i=0,1,2,3, \ldots, n-1$ and $0=u_{0}<u_{1}<\ldots<u_{n-1}<u_{n}=1$, the consecutive radius vectors $\mathbf{r}\left(u_{i}, v\right)$ and $\mathbf{r}\left(u_{i+1}, v\right)$ of the pipes in Equation (10) intersect each other relative to their support curve $\mathbf{C}(u)$. The tested vectors $\mathbf{r}\left(u_{i}, v\right)$ and $\mathbf{r}\left(u_{i+1}, v\right)$ can be computed by Equation (10) through $v=0$ and $v=0.5$, namely

$$
\begin{gathered}
\mathbf{r}\left(u_{i}, 0\right)=\mathbf{T}_{2}\left(u_{i}, 0\right)-\mathbf{C}\left(u_{i}\right)=\Upsilon_{C}\left(u_{i}, 0\right)\left[\cos 0 . \mathbf{v}_{1}+\sin 0 . \mathbf{v}_{2}\right] \\
\mathbf{r}\left(u_{i+1}, 0\right)=\mathbf{T}_{2}\left(u_{i+1}, 0\right)-\mathbf{C}\left(u_{i+1}\right)=\Upsilon_{C}\left(u_{i+1}, 0\right)\left[\cos 0 . \mathbf{v}_{1}+\sin 0 . \mathbf{v}_{2}\right] \\
\mathbf{r}\left(u_{i}, 0.5\right)=\mathbf{T}_{2}\left(u_{i}, 0.5\right)-\mathbf{C}\left(u_{i}\right)=\Upsilon_{C}\left(u_{i}, 0.5\right)\left[\cos \pi . \mathbf{v}_{1}+\sin \pi . \mathbf{v}_{2}\right] \\
\mathbf{r}\left(u_{i+1}, 0.5\right)=\mathbf{T}_{2}\left(u_{i+1}, 0.5\right)-\mathbf{C}\left(u_{i}\right)=\Upsilon_{C}\left(u_{i+1}, 0.5\right)\left[\cos \pi . \mathbf{v}_{1}+\sin \pi . \mathbf{v}_{2}\right] .
\end{gathered}
$$

To control the needed length of the radius vectors $\mathbf{r}\left(u_{i}, 0\right)$ and $\mathbf{r}\left(u_{i}, 0.5\right)$ can use the formulas

$$
\begin{gathered}
\mathbf{r}\left(u_{i}, 0\right)= \pm \lambda\left[\mathbf{T}_{2}\left(u_{i}, 0\right)-\mathbf{C}\left(u_{i}\right)\right]= \pm \lambda \Upsilon_{C}\left(u_{i}, 0\right)\left[\cos 0 . \mathbf{v}_{1}+\sin 0 . \mathbf{v}_{2}\right] \\
\mathbf{r}\left(u_{i}, 0.5\right)= \pm \lambda \mathbf{T}_{2}\left(u_{i}, 0.5\right)-\mathbf{C}\left(u_{i}\right)= \pm \lambda \Upsilon_{C}\left(u_{i}, 0.5\right)\left[\cos \pi \cdot \mathbf{v}_{1}+\sin \pi \cdot \mathbf{v}_{2}\right]
\end{gathered}
$$

with $\lambda$ a real scalar.

Using the position differences test between the end points of the radius vectors that are defined by the position vectors $\mathbf{C}\left(u_{i}\right), \mathbf{T}_{2}\left(u_{i}, 0\right), \mathbf{T}_{2}\left(u_{i}, 0.5\right), \mathbf{C}\left(u_{i+1}\right), \mathbf{T}_{2}\left(u_{i+1}, 0\right)$, and $\mathbf{T}_{2}\left(u_{i+1}, 0.5\right)$, for $i=0,1,2,3, \ldots, n-1$, we can determine $\lambda$ to repair the tube surface shapes, i.e., the pipe surfaces of Equation (10) will not be self intersect. The consecutive radius vectors $\mathbf{r}\left(u_{i}, 0\right)$ 
and $\mathbf{r}\left(u_{i+1}, 0\right)$ will intersect (Figure $\left.2 \mathrm{~b}\right)$, if the angle $\varphi_{2} \geq \varphi_{1}$ in which both angles $\varphi_{1}$ and $\varphi_{2}$ are defined by $\varphi_{1}=\left(\left[\mathbf{C}\left(u_{i+1}\right)\right.\right.$ $\left.\left.\mathbf{C}\left(u_{i}\right)\right], \mathbf{r}\left(u_{i}, 0\right)\right), \varphi_{2}=\left(\left[\mathbf{C}\left(u_{i+1}\right)-\mathbf{C}\left(u_{i}\right)\right],\left[\left(\mathbf{C}\left(u_{i+1}\right)-\mathbf{C}\left(u_{i}\right)\right)+\mathbf{r}\left(u_{i+1}, 0\right)\right]\right)$, and

$$
\begin{gathered}
\varphi_{1}=\arccos \left(\frac{\left[\mathbf{C}\left(u_{i+1}\right)-\mathbf{C}\left(u_{i}\right)\right] \cdot \mathbf{r}\left(u_{i}, 0\right)}{\left\|\left[\mathbf{C}\left(u_{i+1}\right)-\mathbf{C}\left(u_{i}\right)\right]\right\|\left\|\mathbf{r}\left(u_{i}, 0\right)\right\|}\right) \\
\varphi_{2}=\arccos \left(\frac{\left[\mathbf{C}\left(u_{i+1}\right)-\mathbf{C}\left(u_{i}\right)\right] \cdot\left[\left(\mathbf{C}\left(u_{i+1}\right)-\mathbf{C}\left(u_{i}\right)\right)+\mathbf{r}\left(u_{i+1}, 0\right)\right]}{\left\|\left[\mathbf{C}\left(u_{i+1}\right)-\mathbf{C}\left(u_{i}\right)\right]\right\|\left\|\left[\left(\mathbf{C}\left(u_{i+1}\right)-\mathbf{C}\left(u_{i}\right)\right)+\mathbf{r}\left(u_{i+1}, 0\right)\right]\right\|}\right)
\end{gathered} .
$$

Third, when the curves $\mathbf{C}(u)$ are spiral (Figure 2c), We detect the intersection between $\left[\mathbf{r}\left(u_{i}, 0\right), \mathbf{r}\left(u_{i+1}, 0\right)\right]$, $\left[\mathbf{r}\left(u_{i}, 0.5\right), \mathbf{r}\left(u_{i+1}, 0.5\right)\right]$, and for $i=0,1,2,3, \ldots, n-1$ we evaluate the intersection between each $\mathbf{r}\left(u_{i}, 0\right)$ toward all $\left[\mathbf{T}_{2}\left(u_{i+1}, 0\right)-\mathbf{T}_{2}\left(u_{i}, 0\right)\right]$, and for each $\mathbf{r}\left(u_{i}, 0.5\right)$ toward all $\left[\mathbf{T}_{2}\left(u_{i+1}, 0.5\right)-\mathbf{T}_{2}\left(u_{i}, 0.5\right)\right]$.

To implement this pipe construction procedure for Equation (10), we give the illustrations by using the data as follows. In the plane YOZ, if we determine the cubic Bézier curves with $\left[\mathbf{P}_{0}=\langle 0,-9,15\rangle, \mathbf{P}_{1}=\langle 0,5,0\rangle, \mathbf{P}_{2}=\langle 0,12,19\rangle, \mathbf{P}_{3}=\right.$ $\langle 0,16,7\rangle], \Upsilon_{C}(u, v)=-2+\sin (2 \pi v)+\cos (4 \pi v)$, and the vector $\mathbf{v}_{0}=\langle 0,-10,-10\rangle$, then we obtain Figure $2 \mathrm{~d}$. In the plane $\mathrm{x}$ $-\mathrm{z}=0$, if we give the control points $\left[\mathbf{P}_{o}=\langle 15,-25,15\rangle, \mathbf{P}_{1}=\langle-10,5,-10\rangle, \mathbf{P}_{2}=\langle 20,8,20\rangle, \mathbf{P}_{3}=\langle 12,25,12\rangle\right], \Upsilon_{C}(u, v)=2$ $q(u)$ in which $q(u)$ is of Equation (4d) with $q_{0}=0.75 ; q_{x}=0.5 ; q_{1}=1.25 ; q_{0}^{u}=1 ; q_{1}{ }^{u}=-1 ;$ and $\mathbf{v}_{0}=\langle-25,0,0\rangle$, then Equation (10) will present Figure 2e. Using the quartic Bézier curve $\mathbf{C}(u)$, Figure 2f, and Figure $2 \mathrm{~g}$ are constructed by $Y_{C}(u, v)$ inflate-deflate types, and both the constant vector $\mathbf{v}_{0}=\langle 0,-34,-10\rangle$ and the curve $\mathbf{C}(u)$ in the plane YOZ. Meanwhile, Figure $2 \mathrm{~h}$ is presented by $Y_{C}(u, v)$ of constant value, and $\mathbf{v}_{0}=\langle 0,-34,-10\rangle$ outer of that plane. Therefore, we can conclude that Equation (10) is applicable for modeling the constant and the inflate-deflate plane pipes of long measure.

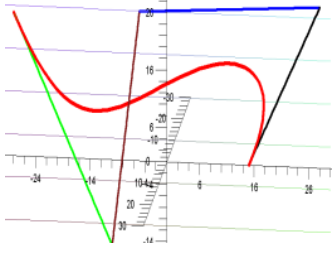

(a) Fluctuative

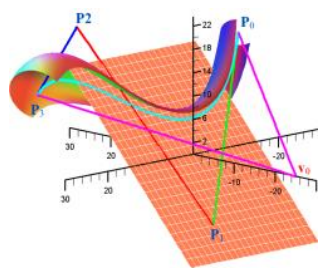

(e)

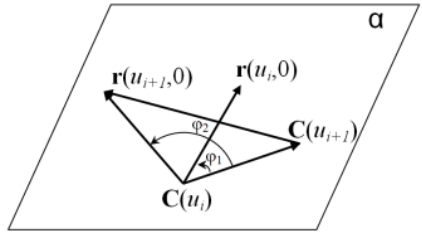

(b) Intersection test

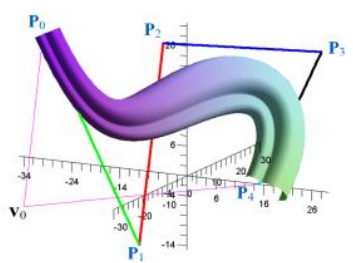

(f)

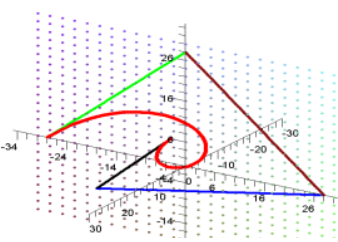

(c) Spiral

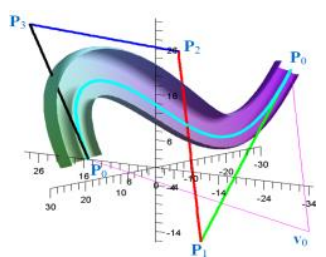

(g)

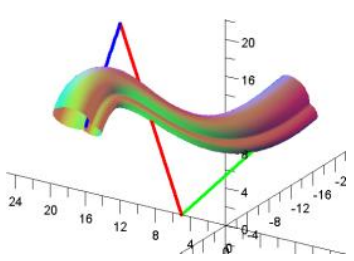

(d) Curve $\mathbf{C}(u)$ in plane $\mathrm{YOZ}$

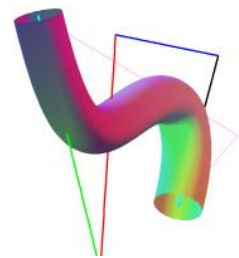

(h)

Figure 2. Pipe profils with their center curves $\mathbf{C}(u)$ in plane

\subsubsection{Case: $\mathbf{C}(u)$ Space Curves}

Consider a regular space curves $\mathbf{C}(u)$ respectively of cubic, quartic Bézier or cubic Hermite curves. We choose a limit that the torsion of curve $\mathbf{C}(u)$ can be tolerated, and $Y_{C}(u, v)$ is defined by circles radius in the planes $\left[\mathbf{v}_{1}, \mathbf{v}_{2}\right]$. The vector $\mathbf{v}_{0}$ fulfills the condition $\left(\mathbf{C}(u)-\mathbf{v}_{0}\right) \neq \mathbf{0}$ and $\mathbf{v}_{0} \neq \mathrm{kt}$ with $\mathrm{k}$ a real scalar for all $0 \leq u \leq 1$. Therefore, the vectors $\mathbf{v}_{2}$ of Equation (9) can be defined for every $u$ in interval $0 \leq \mathrm{u} \leq 1$ and the unit vector $\mathbf{v}_{1}=\mathbf{t} \wedge \mathbf{v}_{2}$ can be computed uniquely.

For many applications, the curves $\mathbf{C}(u)$, generally, must be of arbitrary shapes. To detect the intersection or the crossing over between the consecutive vectors $\mathbf{r}\left(u_{i}, v\right)$ and $\mathbf{r}\left(u_{i+1}, v\right)$ in space, we can use the procedure as follows. For $i=0,1,2,3, \ldots$, $n-1$, the vectors $\left[\mathbf{C}\left(u_{i+1}\right)-\mathbf{C}\left(u_{i}\right)\right]$ and $\mathbf{r}\left(\mathrm{u}_{\mathrm{i}}, v\right)$ determine a plane $\tau_{i}$ (Figure $\left.3 \mathrm{a}\right)$. We project the vector $\mathbf{r}\left(u_{i+1}, v\right)$ to the plane $\tau_{i}$ and we will find the vector $\mathbf{r}_{\mathbf{p}}\left(u_{i+1}, v\right)$ in the form

$$
\mathbf{r}_{\mathbf{p}}\left(u_{i+1}, v\right)=\mathbf{r}\left(u_{i+1}, v\right)-\left[\mathbf{r}\left(u_{i+1}, v\right) \cdot \mathbf{u}\left(u_{i+1}, v\right)\right] \mathbf{u}\left(u_{i+1}, v\right) .
$$

with $\mathbf{u}\left(u_{i+1}, v\right)$ the normal vector of the plane $\tau_{i}$ and $\mathbf{u}\left(u_{i+1}, v\right)=\left(\left[\mathbf{C}\left(u_{i+1}\right)-\mathbf{C}\left(u_{i}\right)\right] \wedge \mathbf{r}\left(u_{i}, v\right)\right) /\left\|\left(\left[\mathbf{C}\left(u_{i+1}\right)-\mathbf{C}\left(u_{i}\right)\right] \wedge \mathbf{r}\left(u_{i}, v\right)\right)\right\|$. Then, we can detect the intersection between $\mathbf{r}\left(u_{i}, v\right)$ and $\mathbf{r}_{\mathbf{p}}\left(u_{i+1}, v\right)$ by using Equation (19). In this case, we have to numerically examine the intersection between the vectors $\mathbf{r}\left(u_{i}, v\right)$ and $\mathbf{r}_{\mathbf{p}}\left(u_{i+1}, v\right)$ in the plane $\tau_{i}$ in every value $v_{\mathrm{i}}$ for $i=0,1,2,3, \ldots, n-1$ and $0=v_{0}<v_{1}<\ldots<v_{n-1}<v_{n}=1$.

When we determine $\mathbf{C}(u)$ quartic Bézier with $\left[\mathbf{P}_{o}=\langle-10,-30,15\rangle, \mathbf{P}_{1}=\langle 0,-32,32\rangle, \mathbf{P}_{2}=\langle 0,30,15\rangle, \mathbf{P}_{3}=\langle 0,-10,5\rangle, \mathbf{P}_{4}=\right.$ $\langle 20,26,-30\rangle], \Upsilon_{C}(u, v)=4, \mathbf{v}_{0}=\langle 30,10,10\rangle$, and $\left[\mathbf{P}_{o}=\langle-10,-30,0\rangle, \mathbf{P}_{1}=\langle 0,-32,32\rangle, \mathbf{P}_{2}=\langle 20,30,15\rangle, \quad \mathbf{P}_{3}=\langle 0,-10,5\rangle\right.$, 
$\left.\mathbf{P}_{4}=\langle 10,26,-30\rangle\right], \Upsilon_{C}(u, v)=q(u)$ in which $q(u)$ is of Equation $(4 \mathrm{~d})$ with $q_{0}=2 ; q_{x}=4 ; q_{1}=3 ; q_{0}{ }^{u}=2 ; q_{1}{ }^{u}=-2, \mathbf{v}_{0}=$ $<8,-16,22>$; we will, respectively, obtain Figure $3 \mathrm{~b}$ and Figure $3 \mathrm{c}$ that present the constant and the inflate-deflate pipes in space. Thus, in general, Equation (10) is also useful for modeling the constant and the inflate-deflate space pipes of long measure with the circle radius in the planes $\left[\mathbf{v}_{1}, \mathbf{v}_{2}\right]$.

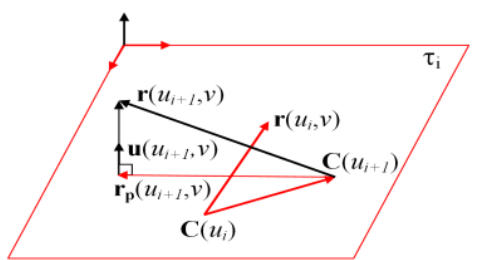

(a)

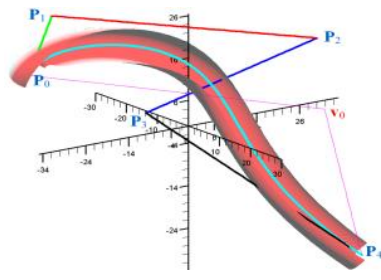

(b)

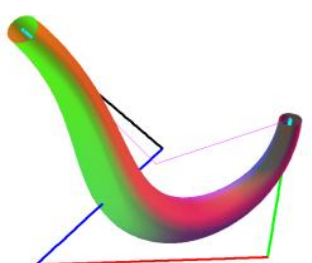

(c)

Figure 3. Pipe profils with their center curves $\mathbf{C}(u)$ in space

\subsection{Construction Test of Short Pipes}

This section discusses the application of Equation (8) with the pipes' center curve $\mathbf{C}(u)$ of the cubic Bézier, the quartic Bézier, and Hermite curve types. To model the short pipes in inflate-deflate and thickness shapes, we restrict the normal unit vectors $\mathbf{n}$ of the curve segment $\mathbf{C}(u)$ such that it has the same sign. For this objectives, we compute the trihedron vectors $[\mathbf{t}, \mathbf{n}, \mathbf{b}]$ of the curves (Figure 4a), then, we present some pipes' design results using Equation (8) as follows.

The second derivative of the cubic and quartic Bézier curves of Equation (11) are respectively

$$
\begin{gathered}
\mathbf{B}_{3}{ }^{\prime \prime}(\mathrm{u})=6\left[\left(\mathbf{P}_{2}-2 \cdot \mathbf{P}_{1}+\mathbf{P}_{\mathrm{o}}\right) \cdot(1-u)+\left(\mathbf{P}_{3}-2 \cdot \mathbf{P}_{2}+\mathbf{P}_{1}\right) \cdot u\right]=\langle\mathrm{Wx}(\mathrm{u}), \mathrm{Wy}(\mathrm{u}), \mathrm{Wz}(\mathrm{u})\rangle \\
\mathbf{B}_{4}{ }^{\prime \prime}(\mathrm{u})=12\left[\left(\mathbf{P}_{2}-2 \mathbf{P}_{1}+\mathbf{P}_{\mathrm{o}}\right)(1-u) \cdot(1-u)+2\left(\mathbf{P}_{3}-2 \mathbf{P}_{2}+\mathbf{P}_{1}\right)(1-u) \cdot u+\left(\mathbf{P}_{4}-2 \mathbf{P}_{3}+\mathbf{P}_{2}\right) \cdot u^{2}\right]
\end{gathered}
$$

with $W_{x}(u)=6\left[\left(P_{2 x}-2 P_{1 x}+P_{o x}\right) \cdot(1-u)+\left(P_{3 x}-2 P_{2 x}+P_{1 x}\right) \cdot u\right] ; W_{y}(u)=6\left[\left(P_{2 y}-2 P_{1 y}+P_{o y}\right) \cdot(1-u)+\left(P_{3 y}-2 P_{2 y}+P_{1 y}\right) \cdot u\right]$; and $W_{z}(u)$ $=6\left[\left(P_{2 z}-2 P_{1 z}+P_{o z}\right) \cdot(1-u)+\left(P_{3 z}-2 P_{2 z}+P_{1 z}\right) \cdot u\right]$.

Using Equation (6a) and Equation (6b) can be determined the normal unit vector $\mathbf{n}_{\mathrm{B} 3}$ and the unit binormal vector $\mathbf{b}_{\mathrm{B} 3}$ of the cubic Bézier curve $\mathbf{B}_{3}(\mathrm{u})$ in the form

$$
\begin{gathered}
\mathbf{n}_{\mathrm{B} 3}=\left\langle\left(M_{x}(u) / s_{o}\right),\left(M_{y}(u) / s_{o}\right),\left(M_{z}(u) / s_{o}\right)\right\rangle \\
\mathbf{b}_{\mathrm{B} 3}=\left\langle\left[R_{y}(u) \cdot M_{z}(u)-M_{y}(u) \cdot R_{z}(u)\right] /\left(s-s_{o}\right),\left[M_{x}(u) \cdot R_{z}(u)-R_{x}(u) \cdot M_{z}(u)\right] /\left(s-s_{o}\right),\left[R_{x}(u): M_{y}(u)-M_{x}(u): R_{y}(u)\right] /\left(s-s_{o}\right)\right\rangle
\end{gathered}
$$

with $\mathrm{s}_{\mathrm{o}}=\left[M_{x}^{2}(u)+M_{y}{ }^{2}(u)+M_{z}{ }^{2}(u)\right]^{1 / 2} ; M_{x}(u)=\left[s^{2} \cdot W_{x}(u)-\left[R_{x}^{2}(u) \cdot W_{x}(u)+R_{x}(u) \cdot R_{y}(u) \cdot W_{y}(u)+R_{x}(u) \cdot R_{z}(u) \cdot W_{z}(u)\right]\right] /\left(s^{4}\right)$; $M_{y}(u)=\left[s^{2} \cdot W_{y}(u)-\left[R_{y}(u) \cdot R_{x} \cdot W_{x}(u)+R_{y}{ }^{2}(u) \cdot W_{y}(u)+R_{y}(u) \cdot R_{z}(u) \cdot W_{z}(u)\right]\right] /\left(s^{4}\right) ; M_{z}(u)=\left[s^{2} \cdot W_{z}(u) \cdot\left[R_{z}(u) \cdot R_{x}(u) \cdot W_{x}(u)+\right.\right.$ $\left.\left.R_{z}(u) \cdot R_{y}(u) \cdot W_{y}(u)+R_{z}^{2}(u) \cdot W_{z}(u)\right]\right] /\left(s^{4}\right)$.

The calculation method of the normal unit vector $\mathbf{n}_{\mathrm{B} 4}$ and the unit binormal vector $\mathbf{b}_{\mathrm{B} 4}$ of the quartic Bézier curve $\mathbf{B}_{4}(\mathrm{u})$ are the same as the cubic Bézier curve $\mathbf{B}_{3}(\mathrm{u})$. Base on equation (8), the inflate-deflate and the thickness cubic and quartic Bézier tubular patches respectively can be written by

$$
\begin{aligned}
& \mathbf{T}_{\mathrm{B} 3}(u, v)=\mathbf{B}_{3}(u)+\Upsilon_{\mathrm{B} 3}(u, v) \cdot\left[\cos (\varphi) \mathbf{b}_{\mathrm{B} 3}+\sin (\varphi) \mathbf{n}_{\mathrm{B} 3}\right] \\
& \mathbf{T}_{\mathrm{B} 4}(u, v)=\mathbf{B}_{4}(u)+\Upsilon_{\mathrm{B} 4}(u, v) \cdot\left[\cos (\varphi) \mathbf{b}_{\mathrm{B} 4}+\sin (\varphi) \mathbf{n}_{\mathrm{B} 4}\right] .
\end{aligned}
$$

The radii of the pipes $\Upsilon_{\mathrm{B} 3}(u, v)$ or $\Upsilon_{\mathrm{B} 4}(u, v)$ are defined by $\Upsilon(u, v)=\rho(u) \cdot r(v)$ with $\varphi=2 \pi v$ and $0 \leq u, v \leq 1$.

On the other hand, the second derivative of the Hermite curves of Equation (14) is

Therefore we obtain

$$
\mathbf{H}^{\prime \prime}(u)=\mathbf{H}_{o}(12 u-6)+\mathbf{H}_{1}(-12 u+6)+\mathbf{H}_{o}^{u}(6 u-4)+\mathbf{H}_{1}^{u}(6 u-2)=\left\langle Z_{x}(u), Z_{y}(u), Z_{z}(u)\right\rangle .
$$

$$
\begin{gathered}
\mathbf{n}_{H}=\left\langle S_{x}(u) / n_{o}, S_{y}(u) / n_{o}, S_{z}(u) / n_{o}\right\rangle \\
\mathbf{b}_{H}=\left\langle\left[N_{y}(u) \cdot S_{z}(u)-S_{y}(u) \cdot N_{z}(u)\right] /\left(h \cdot n_{o}\right),\left[S_{x}(u) \cdot N_{z}(u)-N_{x}(u) \cdot S_{z}(u)\right] /\left(h \cdot n_{o}\right),\left[N_{x}(u) \cdot S_{y}(u)-S_{x}(u) \cdot N_{y}(u)\right] /\left(h \cdot n_{o}\right)\right\rangle
\end{gathered}
$$

with $n_{o}=\left[S_{x}^{2}(u)+S_{y}^{2}(u)+S_{z}^{2}(u)\right]^{1 / 2} ; S_{x}(u)=\left[h^{2} \cdot Z_{x}(u)-\left[N_{x}^{2}(u) \cdot Z_{x}(u)+N_{x}(u) \cdot N_{y}(u) \cdot Z_{y}(u)+N_{x}(u) \cdot N_{z}(u) \cdot Z_{z}(u)\right]\right] / h^{4} ; S_{y}(u)=$ $\left[h^{2} \cdot Z_{y}(u)-\left[N_{y}(u) \cdot N_{x} \cdot Z_{x}(u)+N_{y}^{2}(u) \cdot Z_{y}(u)+N_{y}(u) \cdot N_{z}(u) \cdot Z_{z}(u)\right]\right] / h^{4} ; \quad S_{z}(u)=\left[h^{2} \cdot Z_{z}(u)-\left[N_{z}(u) \cdot N_{x}(u) \cdot Z_{x}(u)+N_{z}(u) \cdot N_{y}(u) \cdot Z_{y}(u)\right.\right.$ $\left.\left.+N_{z}^{2}(u) \cdot Z_{z}(u)\right]\right] / h^{4}$.

Thus the cubic Hermite tubular patches can be expressed by

$$
\mathbf{T}_{H}(u, v)=\mathbf{H}(u)+\Upsilon_{H}(u, v)\left(\cos \varphi \mathbf{b}_{H}+\sin \varphi \mathbf{n}_{H}\right)
$$

with $Y_{H}(\mathrm{u}, \mathrm{v})=\rho(u) \cdot r(v)$, the value $\varphi=2 \pi v$ and $0 \leq \mathrm{u}, \mathrm{v} \leq 1$.

For the application test, we evaluate these equations using the Bézier curve of Equation (11a) with control points $\left[\mathbf{P}_{o}=\right.$ $\left.\langle 22,18,15\rangle, \mathbf{P}_{1}=\langle 8,2,15\rangle, \mathbf{P}_{2}=\langle 18,3,16\rangle, \mathbf{P}_{3}=\langle 25,14,10\rangle\right]$. The functions $\Upsilon_{\mathrm{B} 3}(u, v)$ are defined by $\rho(u)$ of the quartic 
Hermite curve of Equation (4d) with $\left[q_{o}=2, q_{x}(0.3)=2, q_{1}=2, q_{o}{ }^{u}=1, q_{1}{ }^{u}=-1\right], \quad\left[q_{o}=3, q_{x}(0.3)=2, q_{1}=1, q_{o}{ }^{u}=1, q_{1}{ }^{u}\right.$ $=-1]$ and the cubic Bézier of Equation (4b) with $\left[P_{o}=2, P_{1}=1, P_{2}=1, P_{3}=2\right]$, meanwhile, the functions $r(v)$ are in the form $r(v)=4, r(v)=2+\cos (6 . \pi v), r(v)=r_{5}(v)$ with $n=4$ and $r(v)=0.55[5+1 / 20 \cdot \cos (8 \pi v)+\sin (14 \pi v)]$. Using equation $(24)$ will find the pipe patch shapes respectively in Figure 4b, 4c, 4d, 4e, 4f, 4g, 4h and 4i.

On the other hand, when we determine the cubic Hermite curve $\mathbf{H}(u)$ of the equation (14) with the values $\left[\mathbf{H}_{\mathrm{o}}=\langle 45,0,0\rangle\right.$, $\left.\mathbf{H}_{1}=\langle 45,0,15\rangle, \mathbf{H}_{o}^{u}=3 .\langle 15,4,15\rangle, \mathbf{H}_{1}^{u}=3 .\langle 15,4,15\rangle\right]$ and the functions $\Upsilon_{\mathrm{H}}(u, v)$ are defined by $\rho(u)=4$ and equation (4b) of the values $\left[P_{o}=2, P_{1}=0.3, P_{2}=1, P_{3}=2\right]$, the function $r(v)$ in the form $r(v)=15[-2+\sin (2 \pi v)-\cos (4 \pi v)], r(v)=$ $4[-2 \cos (4 \pi v)-\cos (8 \pi v)], r(v)=9 \cdot \cos (4 \pi v)$ and $r(v)=5+1 / 20 \cdot \cos (8 \pi v)+\sin (14 \pi v)$, then equation (28) will present the pipe patches shapes as shown in Figure $4 \mathrm{j}, 4 \mathrm{k}$ and 41.

Based on these results, Equation (24), (25) and Equation (28) can address to construct the pipes in different curvature, thickness (Figure 4e,f,h,i,l) and in different form of volume (Figure 4d,f,k,l). So, they are handy to design various volume fluctuations of the pipes, multiple thickness, and useful to model the inflate-deflate pipe parts.
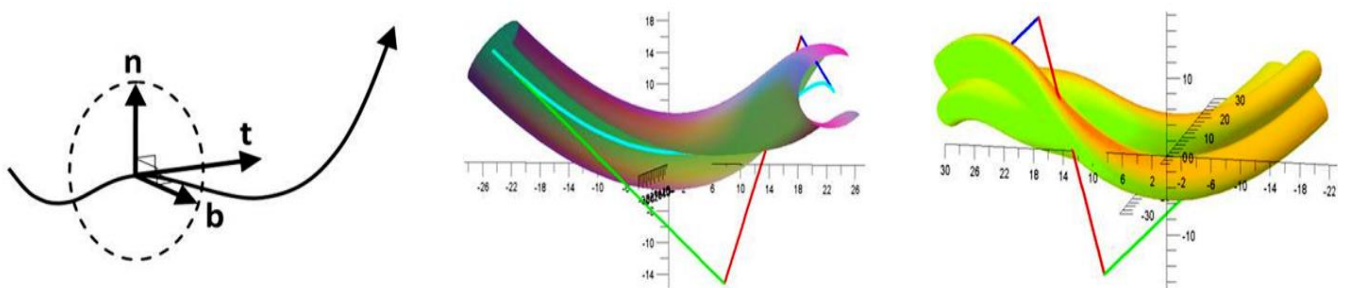

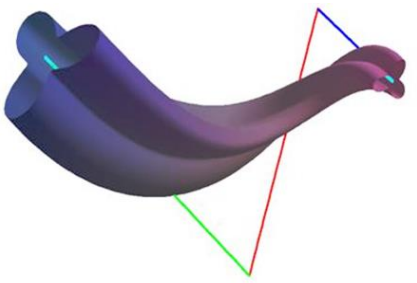

(d)

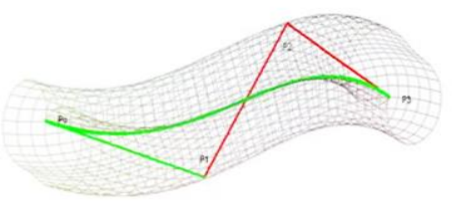

(g)

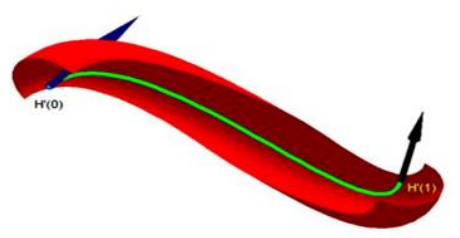

(j)

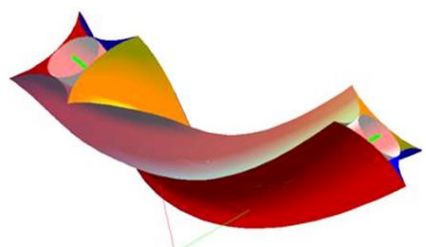

(e)

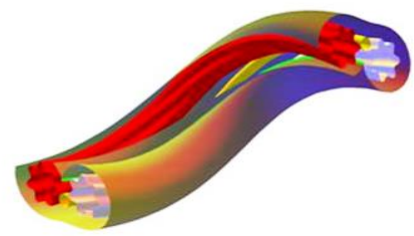

(h)

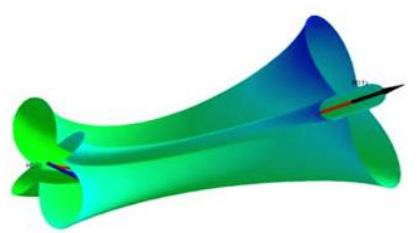

(k)

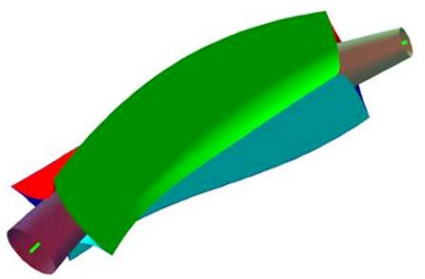

(f)

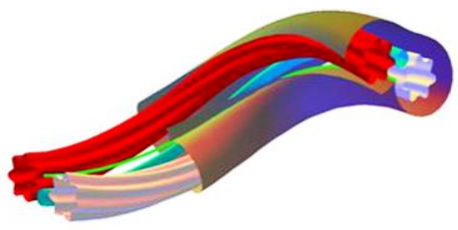

(i)

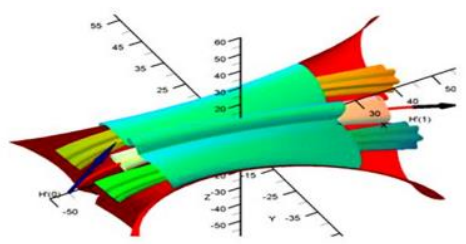

(1)

Figure 4. Inflate-deflate and thickness of short pipe patches modeling

\section{Conclusions}

We have formulated the equations that can be used to model the long pieces and short pices of pipe, and the inflate-deflate pipe patches with its center curves in the form of cubic, quartic Bézier and cubic Hermite curves. These formulas offer design parameters to manipulate the desired shapes of the pipes, i.e., they are handy to design multiple thicknesses, various volume fluctuations of the pipes, and useful to model the inflate-deflate pipe parts. Therefore, by using the presented formulae, it is expected that the construction of any pipe parts will be more effective and satisfied.

The pipeline construction methods have been presented. In the future works, we need to develop how to combine and how to connect small parts of pipes to obtain the length and the branches topology of the pipes. 


\section{Acknowledgments}

The author thanks for the support of the Mathematics staff of Jember University in finishing this research.

\section{References}

Bhatt, A. D., Goel, A., Gupta, U., \& Awasthi, S. (2015). Reconstruction of branched surfaces: Experiments with disjoint b-spline surface. Comput.-Aided Des. \& Appl., 12, 76-85. https://doi.org/10.1080/16864360.2014.949577

Bizzarri, M., Lávička, M., \& Vršek, J. (2015). Canal surfaces with rational contour curves and blends by passing the obstacles. Comput. Aided Des., 64, 55-67. http://doi.org/10.1016/j.cad.2015.03.002

Carmo, M. P. D. (1976). Differential geometry of curves and surface. Prentice Hall Inc Englewood Cliffs, New Jersey.

Fok, S. C., Tan, F. L., \& Leow, K. K. (2012). CAD modelling of solidification phase front around a vertical finned tube. Int. J. Comput. Aided Eng. and Technol., 4, 420-431. https://doi.org/10.1504/IJCAET.2012.048839

Henderson, D. W. (1998). Differential geometry a geometric introduction. Prentice Hall, New Jersey.

Kreyzig, E. (1991). Differential geometry. Dover Publications Inc, New York.

Kusno. (2010). Geometri rancang bangun. Jember University Press, Indonesia.

Kusno, \& Cahyo, A. (2018). Continuous connection of two adjacent pipe parts defined by line, Bézier and Hermite center curves. J. of Phys.: Conf. Ser., 1008. https://doi.org/10.1088/1742-6596/1008/1/012005

Kusno, (2019). On the modeling of the cross section and the longitudinal section pipes. J. of Phys.: Conf. Ser. (Submitted).

Lipschultz, M. (1969). Theory and problems of differential geometry. Schaum's Outline Series, McGraw-Hill, New York.

Lü, R., \& Pottmann, H. (1996). Pipe surfaces with rational spine curve are rational. Comput. Aided Geom. Des., 13, 621-628. https://doi.org/10.1016/0167-8396(95)00051-8

Maekawa, T., Patrikalakis, N. M., Sakkalis, T., \& Yu, G. (1998). Analysis and applications of pipe surfaces. Comput. Aided Geom. Des., 15, 437-458. https://doi.org/10.1016/S0167-8396(97)00042-3

Malaček, K., \& Šibrava, Z. (2006). Blending circular pipes with a cyclic surfaces. J. for Geom. and Garph. 10, 99-107.

Moon, P. H. (2009). Equivolumetric tubular solids for volume-preserving bend of cylinders. Comput. Aided Geom. Des., 26, 137-151. https://doi.org/10.1016/j.cagd.2008.07.002

Moritani, R., Kanai, S., Date, H., Watanabe, M., Nakano, T., \& Yamauchi, Y. (2018). Cylinder-based simultaneous registration and model fitting of laser-scanned point clouds for accurate as-built modeling of piping system. Comput.-Aided Des.\& Appl., 15, 720-733. https://doi.org/10.1080/16864360.2018.1441239.

Mortenson, M. E. (1996). Geometry modeling (2nd Ed). Wiley Computer Publishing, Jonh Wiley and Sons Inc., New York.

Obradovic, R., Popkonstantinovic, B., \& Beljin, B. (2012). Algorithm for approximation transitional developable surfaces between two polygons. Tech. Technol. Educ. Manag., 7, 1907-1913.

Öztürk, G., Byram, B. K., \& Arslan, K. (2010). On canal surfaces in E3. Selçuk. J. of Appl. Math., 11, $103-108$.

Su, Z., Yang, X., Liu, G., Li, W., \& Weiqing, W. (2015). Topology authentication for piping isometric drawings. Comput. Aided Des., 66, 33-44. https://doi.org/10.1016/j.cad.2015.04.005

Zawidski, M., \& Nishinari, K. (2013). Modular pipe-z system for three-dimentional knots. J. for Geom. and Graph., 17, 81-87.

Zhou, P., \& Qian, W. H. (2013). Constructive $\mathrm{G}^{1}$ connection of multiple freeform pipes in arbitrary poses. Comput. Aided Geom. Des., 30, 462-475. https://doi.org/10.1016/j.cagd.2013.02.006

\section{Copyrights}

Copyright for this article is retained by the author(s), with first publication rights granted to the journal.

This is an open-access article distributed under the terms and conditions of the Creative Commons Attribution license (http://creativecommons.org/licenses/by/4.0/). 